\title{
Drug-susceptibility Patterns of Mycobacterium tuberculosis in Mpumalanga Province, South Africa: Possible Guiding Design of Retreatment Regimen
}

\author{
E. Green ${ }^{1,2}$, C.L. Obi ${ }^{3}$, M. Nchabeleng ${ }^{4}$, B.E. de Villiers ${ }^{4}$, P.P. Sein ${ }^{4 *}$,T. Letsoalo ${ }^{4}$, \\ A.A. Hoosen ${ }^{4}$, P.O. Bessong' ${ }^{\prime}$, and R.N. Ndip ${ }^{2,5}$

\begin{abstract}
'AIDS Virus Research Laboratory, Department of Microbiology, University of Venda, South Africa,
2Department of Biochemistry and Microbiology, University of Fort Hare, Private Bag XI3 14, Alice 5700, South Africa,

${ }^{3}$ Research and Academic Directorate,Walter Sisulu University, Mthatha, South Africa, ${ }^{4}$ Deparment of Medical Microbiology,
\end{abstract} \\ University of Pretoria, South Africa (*Deceased), and ${ }^{5}$ Department of Biochemistry and Microbiology, University of Buea, Cameroon
}

\begin{abstract}
Multidrug-resistant tuberculosis (MDR-TB) has been a cause of concern in both developed and developing countries. The prevalence of drug resistance in Mycobacterium tuberculosis (MTB) isolates ( $\mathrm{n}=692)$ from Mpumalanga province was assessed. In total, 692 (64\%) MTB strains from cases with pulmonary TB were tested for susceptibility against rifampicin, isoniazid, ethambutol, and streptomycin using the MGIT 960 instrument. Two hundred and nine (30.2\%) strains were resistant to one or more drugs. Resistance to one drug ranged from $1.4 \%$ for ethambutol to $17.7 \%$ for rifampicin. The prevalence of MDR-TB ranged from $6.7 \%$ for three drugs to $34 \%$ for four drugs, with significant predictors being patients' age-groups of 25-54 years $(\mathrm{p}=0.0012)$ and $>55$ years $(\mathrm{p}=0.007)$. The result showed a high level $(58.4 \%)$ of MDR-TB from cases in Mpumalanga province. To achieve a higher cure rate in this province, drug-susceptibility tests must be done for every case.
\end{abstract}

Key words: Drug resistance, Microbial; Mycobacterium tuberculosis; Risk factors; Tuberculosis; South Africa

\section{INTRODUCTION}

Tuberculosis (TB) remains a deadly infectious disease, affecting millions of people worldwide. Although it is a global epidemic, TB predominantly affects populations in resource-poor countries, where $98 \%$ of all deaths due to TB occur (1). Worldwide, two billion people are latently infected with Mycobacterium tuberculosis (MTB). In 2005, 8.9 million new cases of TB were recorded, and 1.8 million deaths were attributed to the disease $(2,3)$. According to the World Health Organization (WHO), there were an estimated 500,000 cases of MDR-TB in 2007 (4). It is estimated that the actual number of new cases is three times the number of cases reported

Correspondence and reprint requests should be addressed to:

Prof. R.N. Ndip

Department of Biochemistry and Microbiology University of Fort Hare

Private bag X1314

Alice 5700

South Africa

Fax: +27(0) 866224759

Email: rndip@ufh.ac.za OR ndip3@yahoo.com
(5). One-third of 40 million people currently infected with human immunodeficiency virus (HIV) are co-infected with TB $(6,7)$. The changing patterns of global health events over the last two decades, particularly the spread of HIV and drug-resistant TB, have aided in posing serious healthcare problems, thus necessitating the advancement of alternative strategies for effectively combating these re-emerging problems. Strains of MTB resistant to anti-mycobacterial agents, including isoniazid (INH) and rifampicin (RIF), with or without resistance to other first-line drugs, have been reported worldwide (8). Recently-described extensively drug-resistant TB (XDR-TB) has been reported in South Africa (9-11).

The WHO recommends standardized TB treatment regimens based on short-course chemotherapy. The anti-TB drug regimen recommended for the treatment of new cases consists of two months of INH, RIF, pyrazinamide (PZA), and ethambutol (EMB) administration, followed by four months of INH and RIF (12). This treatment is usually effective against wild MTB strains that have never been exposed to anti-TB drugs for more than 30 days (13). The most common indicator of drug- 
resistant (DR) and multidrug-resistant tuberculosis (MDR-TB) is a history of incorrect treatment of TB $(14,15)$.

Continuous surveillance of primary (infection with a drug-resistant strain) and secondary drug resistance (selection and growth of resistant population) patterns of MTB is important in assessing the efficacy of chemotherapy regimens used in the past years and in detecting the problems in past treatments (16). Attention has focused on assessing the global burden of MDR-TB and predicting the future threat of this pathogen. Some investigators predict a global pandemic of MDR-TB whereas others see it as a local problem that can be managed by properly implementing the currently-recommended strategies (14). Despite this attention, there is little consensus on either the magnitude of the problem or its future trend.

There is a paucity of information in primary drug resistance among MTB strains circulating in Mpumalanga province, South Africa. The present study was, therefore, undertaken to measure the prevalence of DR-TB, including MDR-TB, in Mpumalanga province. These results will contribute to guiding the design of retreatment regimen for DR-TB and MDR-TB in the province.

\section{MATERIALS AND METHODS}

\section{Study population}

One thousand and eighty-four sputum samples were collected from 18 districts in Mpumalanga province, South Africa. The inclusion criteria for collecting sputum specimens from patients were according to the Department of Health guidelines, which include clinically-suspected TB, treatment failure, and retreatment among patients. Patients, aged 15-71, years presenting to different clinics and hospitals and outpatient facilities in Mpumalanga during January 2004-December 2006 were eligible for the study. Informed consent was obtained from all patients before enrollment. The Research and Ethics Committee of the University of Venda and the Department of Health and Welfare, Mpumalanga province, approved the study.

\section{Collection of data and definitions}

Clinical, demographic, and epidemiologic data were collected by interviewing patients. Demographic data included age of patient, gender, region of residence, and history of treatment. The study focused primarily on patients who had positive cultures for TB. Multidrug resistance (MDR) was defined as resistance to at least both INH and RIF $(17,18)$ with or without resistance to other drugs. Monoresistance was defined as resistance exclusively to one of the four first-line anti-TB drugs tested (16). Polyresistance was defined as resistance to two or more of the four first-line anti-TB drugs but not both INH and RIF.

\section{Laboratory methods}

Three sputum samples for acid-fast bacillus (AFB) smear microscopy and culture were obtained from each patient enrolled in the study. Specimens were obtained at each district and then transported to TB Laboratory of Dr George Mukhari Hospital in Pretoria where AFB cultures were performed in the BacT/Alert 3D system (BA, bioMérieux, Durham, USA). Dr George Mukhari Hospital is a 1,200-bed tertiary teaching and referral hospital attached to the Medunsa Campus of the University of Limpopo. Sputum specimens are routinely sent to Microbiology Laboratory of Dr George Mukhari Hospital for analysis of TB. Positive cultures were stained by the Ziehl-Neelsen technique. If more than one culturepositive specimen per patient were processed, only one positive result was registered for the patient; similarly, if several negative cultures per patient were encountered, without a positive finding, only one negative result was recorded. Confirmation of MTB was done using the AccuProbe DNA hybridization assay (GenProbe) following the instructions of the manufacturer. All positive cultures were advanced for susceptibility testing.

\section{Susceptibility tests}

Susceptibility to all culture-positive MTB strains was done for the first-line anti-TB drugs, including INH, RMP, and EMB and one second-line drug SM using the MGIT 960 instrument (Becton Dickinson Microbiology Systems, Sparks, MD, USA) (19). The concentrations of drugs were $0.2,40.0,2.0$, and 4.0 $\mu \mathrm{g} / \mathrm{mL}$ for INH, RMP, EMB, and SM respectively. Internal controls were used for ensuring the quality of drug-susceptibility test.

A standard questionnaire and the register were used for determining the history of previous antiTB drug therapy, and the patients were categorized as having either initial or secondary resistance, according to the standard definitions (18). Resistance to INH and RIF with or without resistance to other anti-TB drugs was defined as multidrug resistance.

\section{Statistical analysis}

All statistical analyses were performed using the SPSS software (version 16.0) and the Epi Info soft- 
ware (version 3.4.1). Risk factors for having nonmultidrug resistance and multidrug resistance among culture-confirmed TB cases were assessed. Univariate analysis was performed to determine unadjusted association of TB drug resistance with demographic characteristics of patients. The prevalence ratios (PRs) with 95\% confidence interval (CI) were calculated using StatCalc on Epi Info. A p value of $\leq 0.05$ was considered statistically significant.

\section{RESULTS}

\section{Characteristics of patients}

Of the 1,084 sputum specimens received, 692 (63.8\%) were positive for MTB culture. Drug-susceptibility test and demographic data of the 692 strains were analyzed (Table 1 ). The results showed that $209(30.2 \%)$ strains were resistant to at least one or more drugs. Of the 209 resistant strains, 100 $(47.8 \%)$ were isolated from female patients with a mean age of 35.08 years while 109 (52.2\%) were from males with a mean age of 36.70 years. Seventy-two (34.4\%) patients were hospitalized, 137 (65.5\%) were outpatients, and eight (3.8\%) outpatients were prisoners. Having a positive culture for M. tuberculosis was associated with male gender (Table 1).

\section{Risk of drug-resistant tuberculosis}

The prevalence of different patterns of resistance is shown in Table 2. Overall, 209 strains were resistant to one or more drugs. The proportion of new TB cases with resistance to a single drug ranged from $1.4 \%$ for $\mathrm{EMB}$ to $17.7 \%$ for INH, with a median value of $4.5 \%$. MDR-TB showed a prevalence range of $6.7 \%$ for $\mathrm{INH}+\mathrm{RIF}+\mathrm{EMB} / \mathrm{SM}$ to $34 \%$ for $\mathrm{INH}+\mathrm{RIF}+\mathrm{SM}+\mathrm{EMB}$, with a median of $14.6 \%$. The prevalence of polyresistance to anti-TB drugs tested ranged from $0.5 \%$ for RIF+SM to $6.7 \%$ for INH+SM+EMB (a median of $0.7 \%$ ) (Table 2). The overall resistance to drugs tested ranged from $28 \%$ for INH to $88 \%$ for $\mathrm{EMB}$, with a median of $61 \%$.

There was no significant difference between the MDR-TB cases and the non-MDR-TB cases (49\%) $(\mathrm{p}=0.6)$ in male and female cases respectively (Table 3). A slightly higher prevalence (54\%) of MDR-TB cases was observed in males than in females (46\%). However, for the non-MDR-TB cases, a slightly higher prevalence $(51 \%)$ was observed in females than in males (49\%) (Table 3). The significant predictor of MDR-TB in univariate analysis included patients aged 35-54 years (prevalence ratio $[\mathrm{PR}]=1.84,95 \%$ CI 1.37-2.46, $\mathrm{p}$ value for trends $=0.0012$ ) and patients aged $>54$ years $(\mathrm{PR}=1.55,95 \%$ CI 1.14-2.09, $\mathrm{p}$ value for trends $=0.007$ ) (Table 3).

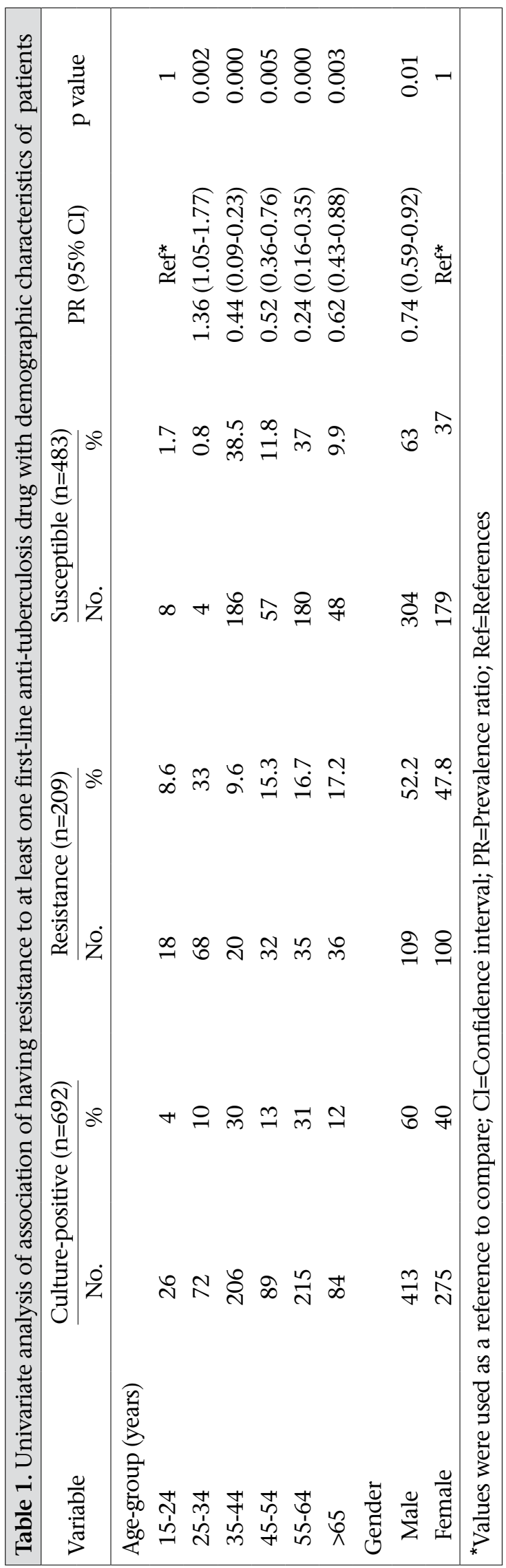




\begin{tabular}{|lcc|}
\hline \multicolumn{1}{|c|}{$\begin{array}{c}\text { Table } 2 \text { Prevalence of first-line anti-tuberculosis } \\
\text { resistance of } 209 \text { Mycobacterium } \\
\text { sis strains from Mpumalanga province }\end{array}$} \\
\cline { 2 - 3 } Resistance profile & No. & $\%$ \\
\hline Total resistance & 209 & 100 \\
Monoresistance & 59 & 28.2 \\
INH only & 37 & 17.7 \\
RIF only & 13 & 6.1 \\
SM only & 6 & 2.9 \\
EMB only & 3 & 1.4 \\
MDR & 122 & 58.4 \\
INH+RIF & 23 & 11 \\
INH+RIF+EMB & 14 & 6.7 \\
INH+RIF+SM & 14 & 6.7 \\
INH+RIF+SM+EMB & 71 & 34 \\
Polyresistance* & 28 & 13.4 \\
INH+SM+EMB & 14 & 6.7 \\
INH+SM & 11 & 5.3 \\
RIF+SM & 1 & 0.5 \\
EMB+SM & 2 & 1 \\
Overall resistant to & & \\
INH drug & 204 & 97.6 \\
RIF drugs & 136 & 65.1 \\
SM drugs & 119 & 57 \\
EMB drugs & 104 & 49.8 \\
\hline * & & \\
\hline
\end{tabular}

*Resistant to two or more drugs but not both isoniazid and rifampicin; $\mathrm{EMB}=$ Ethambutol; INH=Isoniazid; MDRTB=Multi-drug-resistant tuberculosis (resistant to at least both isoniazid and rifampicin with or without resistance to other drugs); RIF=Rifampicin; SM=Streptomycin

\section{DISCUSSION}

South Africa is one of the 22 high-burden countries that contribute approximately $80 \%$ of the total global burden of all TB cases (7). The country has the seventh highest incidence of TB in the world. In South Africa, the incidence of TB has increased, in parallel to the increase in the estimated prevalence of HIV among adults during the past 10 years (11), resulting in the increasing recognition of the problems posed to public health by TB. The risk of DR-TB transmission can be reduced by efficient diagnosis and timely treatment of DR-TB patients (10). Thus, the DR status of a patient needs to be confirmed before treatment. Better access to drugsusceptibility test results at the time of diagnosis of TB would facilitate appropriate selection of treat- ment regimens, thereby minimizing the development of DR strains.

The main focus in the twentieth century was to cure $\mathrm{TB}$ and minimize its transmission to other persons (20). The WHO developed treatment regimen categorized according to different clinical presentations. Management of new patients suspected of having contracted DR strains of M. tuberculosis is essentially similar to the treatment of other new patients. Standardized treatment regimen (STR) in South Africa is given to all patients in a population based on the history of drug used in the country and a representative drug-susceptibility testing.

This study indicated monoresistance to all drugs tested. Overall, we found that resistance to INH $(17.7 \%)$ and RIF (6.1\%) was more prevalent than in other agents. The high resistance of MTB to INH and RIF-the two main drugs in TB treatment-is an alarming sign. In other studies, SM and INH have been reported as more common compared to the other first-line drugs $(21,22)$. Other patterns of anti-TB resistance also existed. In our study, resistance to SM and EMB was less prevalent. Resistance to SM may have been selected following previous and regular administration in the treatment of other bacterial diseases. This finding may indicate a risk vis-a-vis the use of WHO category II (CAT II) regimen for retreatment cases in Mpumalanga. About $28.2 \%$ and $13.4 \%$ of the strains were resistant to mono-drug and poly-drugs (non-MDR) respectively. Thus, approximately $41.6 \%$ of the patients in this study needed to have their treatment changed, as the use of standard therapy in mono-DR-TB and poly-DR-TB has a high risk of failure and can lead to the development of MDR$\mathrm{TB}$ in these patients (23).

More than half (58.4\%) of our study patients were infected with MDR strains. This is different from what was obtained in Cuba during 1999-2000 where $0.5 \%$ of MDR-TB was obtained (24). The relationship between history of receiving anti-TB treatment and drug resistance has been clearly described (25). The prevalence of MDR-TB among patients diagnosed with TB was $16.3 \%$ in mid-1990 (26). In a study conducted in Mpumalanga during 2004-2006 showed a marked decrease in the prevalence of MDR-TB, from $1.92 \%$ to $0.7 \%$ (27). The difference could be explained by the criteria used for the inclusion of patients in this study. Our sample was a convenience sample (patients were requested to give the sputum specimen when they were at the clinics or hospitals) while the National Department of Health did a population-based study. The high number of isolated MDR-TB isolates in our study is 


\begin{tabular}{|c|c|c|c|c|c|c|c|c|}
\hline \multirow[t]{2}{*}{ Variable } & \multicolumn{2}{|c|}{$\begin{array}{l}\text { Total resistance } \\
\quad(\mathrm{n}=209)\end{array}$} & \multicolumn{2}{|c|}{$\begin{array}{l}\text { MDR-TB cases } \\
\quad(\mathrm{n}=122)\end{array}$} & \multicolumn{2}{|c|}{$\begin{array}{l}\text { Non-MDR-TB } \\
\text { cases }(\mathrm{n}=87)\end{array}$} & \multirow[t]{2}{*}{ PR $(95 \%$ CI $)$} & \multirow[t]{2}{*}{$\mathrm{p}$ value } \\
\hline & No. & $\%$ & No. & $\%$ & No. & $\%$ & & \\
\hline \multicolumn{9}{|c|}{ Age-group (years) } \\
\hline $15-34$ & 86 & 41 & 36 & 29.5 & 50 & 57 & $10.70-1.42$ & 1.00 \\
\hline $35-54$ & 52 & 25 & 40 & 36 & 12 & 13 & $1.84(1.37-2.46)$ & 0.0012 \\
\hline $55-75$ & 71 & 34 & 46 & 41 & 25 & 29 & $1.55(1.14-2.09)$ & 0.007 \\
\hline \multicolumn{9}{|l|}{ Gender } \\
\hline Male & 109 & 52 & 66 & 54 & 43 & 49 & $1.08(0.86-1.36)$ & 0.6 \\
\hline Female & 100 & 48 & 56 & 46 & 44 & 51 & $10.78-1.28$ & 1.00 \\
\hline
\end{tabular}

of concern in Mpumalanga where the TB-control programme has not been functioning optimally. In general, a high rate of MDR-TB among patients is suggestive of poor management of past and existing disease (27). MDR-TB is associated with higher rates of failure and death, is more difficult and expensive to treat than drug-susceptible TB (7), and has an increased risk of adverse drug-effects for patients (28).

Knowledge-base for future intervention can be provided by identifying risk factors for infection, disease, and MDR-TB $(29,30)$. In our study, the strongest risk factor of having drug resistance was the age-group of 35-44 years and 55-60 years $(\mathrm{p}<$ 0.001). In line with other reports $(31,32)$, the risk of MDR-TB was observed in the age-group of 35-54 years $(\mathrm{p}=0.0012$ at $95 \% \mathrm{CI})$ and $>54$ years $(\mathrm{p}=0.007$ at CI 95\%). These results could be a reflection of the 2005 situation when directly-observed therapy, short-course strategy (DOTS) was interrupted in Mpumalanga (26). Elderly persons had been exposed to the organisms in the past, and during the process of treatment, resistance was acquired while young patients are more likely to have acquired the bacilli more recently when they were more likely to be resistant.

In our study, $\mathrm{TB}$, including MDR-TB, was more common among males than among females. Other researchers also observed this trend. In a systematic review, MDR-TB cases were more likely to be associated with males in Western Europe while, in Eastern Europe, the male gender was not associated with MDR-TB $(31,33)$. It was assumed that the male gender could modify the association between treatment and MDR-TB since men are believed to be less adherent to treatment than women are.

The spread of DR-TB in Mpumalanga could seriously hamper efforts to control TB. Limited resources and laboratory capacity are a few limitations in the proper control of TB. Increasing the laboratory capacity to deal with DR-TB as recommended by the Global Plan to Stop TB and better referral of specimens from patients living in remote areas to regional or national reference laboratories should be encouraged to ensure success of the TB-control programme and control transmission of DR strains.

The study encountered some limitations. One of these was the potential misclassification of new and retreatment cases; some cases registered as new may actually have had treatment for TB in the past. Classification was based on the patient's history of prior treatment for TB and review of medical records (which were available for all but four patients enrolled). Due to this reason, categorizing the cases as new or retreatment was omitted. Our study, carried out at selected sites in Mpumalanga, was not population-based, which can introduce bias. The high rate of missing data (demographic profile) among patients with a negative AFB culture was because the study focused primarily on patients with a positive culture. However, our study provides important continual data on drug resistance in Mpumalanga.

Although the sample size is small, the results suggest that multidrug resistance is likely to pose a significant threat to health and impede the success of TB management in Mpumalanga, if adequate control measures are not implemented. Monitoring the trend of resistance should remain a priority.

\section{ACKNOWLEDGEMENTS}

The study was supported by the National Research Foundation, South Africa. The authors are thankful to the laboratory management for allowing them to work in their departmental laboratory at the University of Limpopo, Medunsa Campus. They thank the staff of the TB section, Microbiological 
Pathology Laboratory, NHLS, Dr George Mukhari Tertiary Laboratory Complex, Pretoria, for technical assistance. The authors dedicate this work to their colleague Dr. P.P. Sein who passed away recently.

\section{REFERENCES}

1. Grange JM, Zumla A. The global emergency of tuberculosis: what is the cause? J R Soc Promot Health 2002;122:78-81.

2. World Health Organization. Global tuberculosis control—surveillance, planning, financing: WHO report 2007. Geneva: World Health Organization, 2007. 270 p. (WHO/HTM/TB/2007.376).

3. World Health Organization. Seventh meeting [of the] Strategic and Technical Advisory Group for Tuberculosis (STAG-TB): report on conclusions and recommendations, 11-13 June 2007, Geneva. Geneva: World Health Organization, 2007. 32 p.

4. World Health Organization. Global tuberculosis control: epidemiology, strategy, financing. Geneva: World Health Organization, 2009. 303 p. (WHO/ HTM/TB/2009.411).

5. Blower SM, Chou T. Modeling the emergence of the 'hot zones': tuberculosis and the amplification dynamics of drug resistance. Nat Med 2004;10:1111-6.

6. Friedland G, Churchyard GJ, Nardell E. Tuberculosis and HIV coinfection: current state of knowledge and research priorities. J Infect Dis 2007;196:S1-3.

7. World Health Organization. Global tuberculosis control 2008: surveillance, planning and financing: WHO report 2008. Geneva: World Health Organization, 2008. 294 p. (WHO/HTM/TB/2008.393).

8. Ani AE, Idoko J, Dalyop YB, Pitmang SL. Drug resistance profile of Mycobacterium tuberculosis isolates from pulmonary tuberculosis patients in Jos, Nigeria. Trans R Soc Trop Med Hygi 2009;103:67-71.

9. Singh JA, Upshur R, Padayatchi N. XDR-TB in South Africa: no time for denial or complacency. PLoS Med 2007; 4:e50.

10. World Health Organization. Report of the meeting of the WHO Global Task Force on XDR-TB. Geneva: World Health Organization, 2006. 23 p. (WHO/HTM/ $\mathrm{TB} / 2006.375)$.

11. Centers for Disease Control and Prevention. Emergence of Mycobacterium tuberculosis with extensive resistance to second-line drugs - worldwide, 20002004. MMWR Morb Mortal Wkly Rep 2006;55:301-5.

12. World Health Organization. Anti-tuberculosis drug resistance in the world. The WHO/IUATLD Global Project on anti-tuberculosis drug resistance surveillance, 1994-1997. Geneva: World Health Organization, 1997. 227 p. (WHO/TB/97.229).
13. Fattorini L, Migliori GB, Cassone A. Extensively drugresistant (XDR) tuberculosis: an old and new threat. Ann Ist Super Sanita 2007;43:317-9.

14. Ait-Khaled N, Enarson DA. Tuberculosis: a manual for medical students. Geneva: World Health Organization, 2003. 141 p. (WHO/CDS/TB/99.272).

15. Cohen T, Sommers B, Murray M. The effects of drug resistance on the fitness of Mycobacterium tuberculosis. Lancet Infect Dis 2003;3:13-21.

16. Velayati AA, Masjedi MR, Mohammadi M. Textbook of tuberculosis. Tehran: Markaz Nashr Daneshgahi, 1977:819-33 [Persian].

17. Crofton J, Chaulet P, Maher D. Guidelines for the management of drug-resistant tuberculosis. Geneva: World Health Organization, 1997. 186 p. (WHO/TB/ 96.210(rev)).

18. Zager EM, McNerney R. Mulridrug-resistant tuberculosis. BMC Infect Dis 2008;8:10.

19. Rüsch-Gerdes S, Pfyffer GE, Casal M, Chadwick M, Siddiqi S. Multicenter laboratory validatation of the BACTEC MGIT 960 technique for testing susceptibilities of Mycobacterium tuberculosis to classical second-line drugs and newer antimicrobials. J Clin Microbiol 2006;44:688-92.

20. World Health Organization. Global tuberculosis control: surveillance, planning, financing: WHO report. Geneva: World Health Organization, 2003. 229 p. (WHO/CDS/TB/2003.316).

21. Mdivani N, Zangaladze E, Volkova N, Kourbatova E, Jibuti T, Shubladze et al. High prevalence of multidrug-resistant tuberculosis in Georgia. Int J Infect Dis 2008;12:635-44.

22. Aziz MA, Wright A, Laszlo A, De Muynck A, Portaels F, Van Deun A et al. Epidemiology of antituberculosis drug resistance (the Global Project on Anti-tuberculosis Drug Resistance Surveillance): an updated analysis. Lancet 2006;368:2142-54.

23. Blumberg HM, Burman WJ, Chaisson RE, Daley CL, Etkind SC, Friedman LN et al. American Thoracic Society/Centers for Disease Control and Prevention/ Infectious Diseases Society of America: treatment of tuberculosis. Am J Respir Crit Care Med 2003;167:60362.

24. Montoro E, Lemus D, Echemedía M, Aemas L, González-Ochoa E, Llanes MJ et al. Drug-resistant tuberculosis in Cuba. Results of the three global projects. Tuberculosis (Edinb) 2006;86:319-23.

25. Quy HT, Cobelens FGJ, Lan NTN, Buu TN, Lambregts CSB, Borgdorff MW. Treatment outcomes by drug resistance and HIV status among tuberculosis patients in Ho Chi Minh City, Vietnam. Int J Tuberc Lung Dis 2006;10:45-51. 
26. Weyer K, Lancaster J, Balt E, Durrheim D. Tuberculosis drug resistance in Mpumalanga province, South Africa (abstract). Int J Tuberc Lung Dis 1998;2:S165.

27. South Africa. National Department of Health. Tuberculosis strategic plan for South Africa, 2007-2011. TB strategic plan report. Gauteng: National Department of Health, Government of South Africa, 2006. 66 p.

28. Han LL, Sloutsky A, Canale SR, Naroditskaya V, Shin $\mathrm{SS}$, et al. Acquisition of drug resistance in multidrugresistant Mycobacterium tuberculosis during directlyobserved empiric retreatment with standardized regimens. Int J Tuberc Lung Dis 2005;9:818-21.

29. Faustini A, Hall AJ, Perucci CA. Risk factors for multidrug-resistant tuberculosis in Europe: a systemic review. Thorax 2006;61:158-63.

30. Choi JC, Lim Y, Suh GY, Chung MP, Kim H, Kwon OJ et al. Drug resistance rates of Mycobacterium tuberculosis at a private referral center in Korea. J Korean Med Sci 2007;22:677-81.
31. Chan ED, Iseman $M D$. Current medical treatment for tuberculosis. BMJ 2002;325;1282-6.

32. Han LL, Sloutsky A, Canales R, Naroditskaya V, Shin SS, Seung KJ et al. Acquisition of drug resistance in multidrug-resistant Mycobacterium tuberculosis during directly observed empiric retreatment with standardized regimens. Int J Tuberc Lung Dis 2005;9:818-21.

33. Faustini A, Hall AJ, Perucci CA. Risk factors for multidrug-resistant tuberculosis in Europe: a systemic review. Thorax 2006;61:158-63.

34. Choi JC, Lim Y, Suh GY, Chung MP, Kim H, Kwon OJ et al. Drug resistance rates of Mycobacterium tuberculosis at a private referral center in Korea. J Korean Med Sci 2007;22:677-81.

35. Casal M, Vaquero M, Rinder H, Tortoli E, Grosset J, Rüsch-Gerdes $\mathrm{S}$ et al. A case-control study for multidrug-resistant tuberculosis: risk factors in four European countries. Micro Drug Resist 2005;11:62-7. 